DFPD 95/TH/19

\title{
Markov diffusions in comoving coordinates and stochastic quantization of the free relativistic spinless particle
}

\author{
Laura M. Morato \\ Facoltà di Scienze dell' Università di Verona, Via delle Grazie, 37134 Verona, \\ and Dipartimento di Fisica "G. Galilei", Università di Padova, \\ Via Marzolo 8, 35131 Padova, Italy \\ Lorenza Viola \\ Dipartimento di Fisica "G. Galilei", Università di Padova, and INFN, \\ Sezione di Padova, Via Marzolo 8, 35131 Padova, Italy
}

(October 4, 2018)

\begin{abstract}
We revisit the classical approach of comoving coordinates in relativistic hydrodynamics and we give a constructive proof for their global existence under suitable conditions which is proper for stochastic quantization. We show that it is possible to assign stochastic kinematics for the free relativistic spinless particle as a Markov diffusion globally defined on $\mathrm{M}^{4}$. Then introducing dynamics by means of a stochastic variational principle with Einstein's action, we are lead to positive-energy solutions of Klein-Gordon equation. The procedure exhibits relativistic covariance properties.

02.50.-r, 03.65.-w, 03.30.+p
\end{abstract}

Typeset using REVTEX 


\section{INTRODUCTION}

Probability Theory, and Markov processes in particular, are currently recognized as key elements for a proper modelization of various relativistic phenomena within the framework of the Euclidean formulation of Quantum Field Theory. On the other hand, the construction of a mathematically consistent description of diffusive phenomena in Minkowskian spaces must face severe problems. This is due essentially to the difficulty to make the Markovian property compatible with the required relativistic covariance (for different approaches and proposals concerning this longstanding open problem see for example Refs. [1 8 and [9]). A satisfactory theory seems to be still lacking.

The aim of this work is to introduce a new method for treating phenomena described by Markovian diffusions in the configurational space within a relativistic setting. As a first application we procede to perform a covariant stochastic quantization of the free relativistic spinless particle. From a physical point of view, the idea underlying our construction can be sketched as follows. Let us consider, for the sake of simplicity, a three-dimensional Markov diffusion with constant coefficient equal to $\nu$, satisfying Itô's stochastic differential equation

$$
d q(t)=b(q(t), t) d t+\nu^{\frac{1}{2}} d w(t), \quad t \in[0,+\infty),
$$

where $b$ is a drift-field and $w$ a standard Wiener process. Introducing the time dependent density $\rho$ of the process $q(t)$, one can show that under some regularity assumption 10,11 there exists a velocity field $v$ (usually called the "current velocity field") such that the following continuity equation holds:

$$
\partial_{t} \rho+\nabla \cdot(\rho v)=0
$$

Thus, if $b$ is sufficiently smooth, to every Markov process given by a solution of (1.1) can be associated an "hydrodynamical (Eulerian) structure" represented by the time dependent couple $(\rho, v)$, which satisfy the constraint (1.2).

Let us suppose that, together with the diffusion, we are interested to consider, at a generic point $q$ and time $t$, basic observable quantities as energy-momentum tensor, entropy, 
temperature and so on. Thus, in agreement with the approach usually adopted in the relativistic hydrodynamics of a perfect fluid, we would define those objects in the frame of an observer which moves at point $q$ and time $t$ with velocity $v(q, t)$ (the "comoving observer" at point $q$ and time $t$ ). Intuitively, it is therefore reasonable to extend this prescription also to the "probabilistic" objects we are considering. This idea was in fact already exploited in Ref. [12] to show that it is possible to redefine Nelson's stochastic kinematics in a "small" neighborhood of properly defined "comoving observers"; starting from this and a suitable extension of the Einstein action, the Klein-Gordon equation for the free relativistic particle was there derived without ad hoc assumptions.

In the present work we will go beyond the local approach outlined above, and we introduce instead a "global comoving coordinate system". A non linear transformation is performed on flat space from natural coordinates to a class of comoving ones. Our discussion will not depend on the particular choice in this class and the results will be expressed in the language of general curvilinear coordinates. The method is intimately connected with classical results as Frobenius theorem for vector fields and it is close to the construction of cosmic standard coordinates in General Relativity (see for example Ref. [13]). We will give a precise and simple formulation of the basic mathematical facts which does not seem to be commonly available in the literature and which, in particular, is proper for the application to stochastic quantization.

The content of the work is organized as follows: in Sec. II we give a sufficient condition for a vector field $V$ on $\mathrm{R}^{m}, m>1$, induces a "global comoving coordinate system". In Sec. III the same result is reformulated for vector fields on $\mathrm{M}^{4}$ and pseudo-Riemannian manifolds. In Sec. IV we apply these results to redefine Nelson's stochastic kinematics for a free relativistic spinless particle and to derive in a simple and fully consistent mathematical way the Klein-Gordon equation. The stochastic interpretation of different subsets of solutions to this equation is given in Sec. V, where the non relativistic limit is also described. Finally, in Sec. VI we discuss some possible physical implications and briefly consider some future developments and applications. 


\section{VECTOR FIELDS GENERATING GLOBAL COMOVING COORDINATES IN EUCLIDEAN SPACES}

Let us consider a vector field $V: \mathrm{R}^{1+n} \longrightarrow \mathrm{R}^{1+n}, x \mapsto V(x)$. We shall use for the components of $x$ and $V$ the notation $x=\left(x^{0}, x^{1}, \ldots, x^{n}\right)$ and $V=\left(V^{0}, V^{1}, \ldots, V^{n}\right)$. We are searching conditions on $V$ so that there exists a coordinate transformation $\Phi$ which changes the algebric vector $V(x)$ into $V^{\prime}(x):=(\|V(x)\|, 0, \ldots, 0)$ for every $x \in \mathrm{R}^{1+n}$. The inverse function theorem allows to prove the following:

Theorem 1: Let $V$ be a vector field on $\mathrm{R}^{1+n}$ with components $\left(V^{0}, V^{1}, \ldots, V^{n}\right)$, which satisfies the following conditions:

i) $V^{0}(x) \neq 0 \quad \forall x \in \mathrm{R}^{1+n}$,

ii) There exists a scalar field $S \in \mathcal{C}^{r}\left(\mathrm{R}^{1+n}, \mathrm{R}\right), r \geq 2$, such that

$$
V^{\mu}(x)=\frac{\partial}{\partial x^{\mu}} S(x), \quad \forall \mu=0,1, \ldots, n, \forall x \in \mathrm{R}^{1+n} .
$$

Then there exists a diffeomorphism $\Phi: \mathrm{R}^{1+n} \longrightarrow \mathrm{R}^{1+n}$ such that

$$
V^{\prime \mu}(x)=\sum_{\nu=0}^{n} \frac{\partial \Phi^{\mu}(x)}{\partial x^{\nu}} V^{\nu}(x)=\left\{\begin{array}{cl}
\|V(x)\| & \text { if } \mu=0 \\
0 & \text { otherwise }
\end{array}\right.
$$

Proof: Since $V$ is $\mathcal{C}^{1}$, then there exists the congruence $C_{V}$ generated by $V$. That is, given any point $x \in \mathrm{R}^{1+n}$, there exists a unique integral curve $\gamma_{x} \in C_{V}$ to which $x$ belongs. For a fixed $x_{0} \in \mathrm{R}^{1+n}$ let us consider the equation

$$
S(x)-S\left(x_{0}\right)=0
$$

Denoting thus $y_{0}$ the $n$-dimensional vector $\left(x_{0}^{1}, \ldots, x_{0}^{n}\right)$, by assumptions $\left.i\right)$ and $\left.i i\right)$ and the implicit function theorem there exists a neighborhood $I_{y_{0}} \subset \mathrm{R}^{n}$ of $y_{0}$ and a function $f \in \mathcal{C}^{1}\left(I_{y_{0}}, \mathrm{R}\right)$ such that

$$
S\left(f\left(x^{1}, \ldots, x^{n}\right), x^{1}, \ldots, x^{n}\right)-S\left(x_{0}\right)=0 \quad \forall\left(x^{1}, \ldots, x^{n}\right) \in I_{y_{0}} .
$$

Let now $\Gamma$ denote the border of $I_{y_{0}}$ and $\mathrm{p}$ any point belonging to $\Gamma$. Then the $\lim _{\mathrm{q} \rightarrow \mathrm{p}} f(\mathrm{q})$ may be finite or infinite (the existence is ensured by the fact that the application of the 
implicit function theorem in $\mathrm{p}$ would otherwise lead to a contradiction). In the first case one can apply again the implicit function theorem at $\mathrm{p}$, and so on. Thus for each $x_{0} \in \mathrm{R}^{1+n}$ equation (2.1) defines a unique regular $n$-dimensional hypersurface $\Sigma_{x_{0}}$ in $\mathrm{R}^{1+n}$. We also observe that the vector

$$
V(x)=\left(\frac{\partial S(x)}{\partial x^{0}}, \frac{\partial S(x)}{\partial x^{1}}, \ldots, \frac{\partial S(x)}{\partial x^{n}}\right)
$$

is by construction orthogonal to the surface in the point $x$. Hence we can conclude that for each point $x_{0}$ in $\mathrm{R}^{1+n}$ there exists a unique hypersurface $\Sigma_{x_{0}}$ and a unique integral curve $\gamma_{x_{0}}$ to which $x_{0}$ belongs. In addition, $\gamma_{x_{0}}$ is orthogonal to $\Sigma_{x_{0}}$ in $x_{0}$. In other words, the congruence $C_{V}$ is globally hypersurface-orthogonal.

To construct the new coordinate system in $\mathrm{R}^{1+n}$ let us fix a point $O^{\prime}$ as new origin and consider $\gamma_{O^{\prime}}$ (with the natural orientation) and $\Sigma_{O^{\prime}}$. Being $\Sigma_{O^{\prime}}$ a regular hypersurface imbedded in $\mathrm{R}^{1+n}$, it is also a $n$-dimensional Riemannian manifold. Let us introduce on it a coordinate system $q^{1}, \ldots, q^{n}$ (notice that this surface has a unique chart $\mathrm{R}^{n}$ ) and let $\sigma_{i j}, i, j=1, \ldots, n$ be its metric tensor, induced by the Euclidean scalar product in $\mathrm{R}^{1+n}$. Then to every point $x \in \mathrm{R}^{1+n}$ there corresponds a unique point on $\gamma_{O^{\prime}}$ (intersection between $\gamma_{O^{\prime}}$ and $\Sigma_{x}$ ) with coordinate $\lambda(x)$ and a unique point on $\Sigma_{O^{\prime}}$ (intersection of $\Sigma_{O^{\prime}}$ and $\gamma_{x}$ ) with coordinates $q^{1}(x), \ldots, q^{n}(x)$, and viceversa. Therefore the map (see Fig. 1)

$$
\begin{aligned}
\Phi: \quad \mathrm{R}^{1+n} & \longrightarrow \mathrm{R}^{1+n} \\
x & \longmapsto \xi:=\left(\lambda(x), q^{1}(x), \ldots, q^{n}(x)\right)
\end{aligned}
$$

is one-to-one. Existence and continuity of first-order partial derivatives of $\Phi$ immediately follows from the regularity of the integral curves and of the hypersurfaces we are considering.

Let us now denote $\varphi_{x}$ the solution of the ordinary differential equation

$$
\dot{\xi}(\tau)=V(\xi(\tau))
$$

with initial condition 


$$
\varphi_{x}(\tau)=x
$$

Hence we have

$$
\begin{aligned}
d \xi^{\mu}(\tau) & =\frac{\partial \Phi^{\mu}}{\partial x^{\nu}} d \varphi_{x}^{\nu}(\tau)+O\left(\left\|d \varphi_{x}(\tau)\right\|^{2}\right) \\
& =\frac{\partial \Phi^{\mu}}{\partial x^{\nu}} V^{\nu}(x) d \tau+O\left(d \tau^{2}\right) .
\end{aligned}
$$

Let us now choose $\lambda(x)$ equal to the length of the arc on $\gamma_{O^{\prime}}$ and $\xi^{0}:=\lambda(x)$. Then we observe that defining $V^{\prime \mu}$ by the equality

$$
V^{\prime \mu}:=\frac{\partial \Phi^{\mu}}{\partial x^{\nu}} V^{\nu}
$$

we immediately see that, since $d \xi^{\mu}(\tau)=0$ for $\mu=1, \ldots, n$ and $d \xi^{0}(\tau)=\|V(x)\| d \tau+O\left(d \tau^{2}\right)$, only the component $V^{\prime 0}$ is different from zero and it is equal to $\|V(x)\|$.

It is not necessary to particularize the coordinate $\lambda$ to be the length of the arc on $\gamma_{O^{\prime}}$. In fact we can prove the following:

Corollary 1: Let $\xi^{0}=\lambda$ be any coordinate on $\gamma_{O^{\prime}}$. Introducing on $\gamma_{O^{\prime}}$ the positive metric function $f(\lambda)$ we have

$$
V^{\prime \mu}(x)=\left\{\begin{array}{cl}
\frac{\|V(x)\|}{\sqrt{f(\lambda(x))}} & \text { if } \mu=0 \\
0 & \text { otherwise }
\end{array}\right.
$$

Proof: Denoting by $l(\lambda)$ the length of the arc $\left(O^{\prime}, \lambda\right)$, we can rephrase eqs. (2.3)-(2.4) by observing that

$$
d \xi^{0}=d \lambda=\frac{d l(\tau)}{\sqrt{f(\lambda)}}=\frac{\|V(x)\|}{\sqrt{f(\lambda)}} d \tau+O\left(d \tau^{2}\right)=V^{\prime 0}(\xi) d \tau+O\left(d \tau^{2}\right)
$$

from which the result follows.

The coordinate transformation $\Phi$ transforms the Euclidean metric tensor $\delta_{\mu \nu}$ into a new one, which we denote by $g_{\mu \nu}^{E}$. This transformed tensor has in turn a particularily simple structure, as one can immediately recognize by observing that any vector $d x \in \mathrm{R}^{1+n}$ applied 
at the point $x$ can be decomposed into two orthogonal vectors $d z$ and $d y$, where $d z$ is tangent to $\gamma_{x}$ at $x$ and $d y$ is tangent to $\Sigma_{x}$ at $x$. Thus we can write

$$
\begin{aligned}
\|d x\|^{2} & =\|d z\|^{2}+\|d y\|^{2} \\
& =f(\lambda) d \lambda(x)^{2}+\sum_{i, j=1}^{n} \sigma_{i j}\left(q^{1}(x), \ldots, q^{n}(x)\right) d q^{i}(x) d q^{j}(x)+O\left(\|d x\|^{4}\right),
\end{aligned}
$$

where as before $f(\lambda)$ and $\sigma_{i j}$ are the components of the metric tensor of $\gamma_{O^{\prime}}$ and $\Sigma_{O^{\prime}}$ in the given coordinate $\lambda(x)$ and $q^{1}(x), \ldots, q^{n}(x)$ respectively. Thus we can state the following:

Corollary 2: The global coordinate transformation $\Phi: x \mapsto \xi:=\Phi(x), x \in \mathrm{R}^{1+n}$, transforms the Euclidean metric tensor $\delta_{\mu \nu}$ into the tensor $g_{\mu \nu}^{E}$ with components

$$
g_{\mu \nu}^{E}(\xi):=\left\{\begin{array}{cl}
f\left(\xi^{0}\right) & \mu=\nu=0, \\
0 & \mu=0, \nu=1, \ldots, n ; \nu=0, \mu=1, \ldots, n \\
\sigma_{\mu \nu}\left(\xi^{1}, \ldots, \xi^{n}\right) & \text { otherwise } .
\end{array}\right.
$$

Owing to the explicit structure of the transformed metric tensor, we are able to completely characterize our construction from the geometric point of view. In particular, being the map $\Phi$ a global coordinate transformation on flat space $\mathrm{R}^{1+n}$, we know that the Riemann curvature tensor associated to $g^{E}$ identically vanishes. We shall see now that this property extends to $\sigma$, so that $\Sigma_{O^{\prime}}$ eventually has only an extrinsic curvature. We summarize the result in the following:

Corollary 3: The hypersurface $\Sigma_{O^{\prime}}$ has zero intrinsic curvature.

Proof: We need to prove that the $n$-dimensional Riemann curvature tensor $R_{k l m}^{(n) i}$, $i, k, l, m=1, \ldots, n$ associated to $\sigma$ is equal to zero. Starting from the $(1+n)$-dimensional tensor associated to $g^{E}$, let us express it, as usual, in terms of Christoffel symbols:

$$
R_{k l m}^{(1+n) i}=\frac{\partial \Gamma_{k m}^{i}}{\partial \xi^{l}}-\frac{\partial \Gamma_{k l}^{i}}{\partial \xi^{m}}+\Gamma_{\alpha l}^{i} \Gamma_{k m}^{\alpha}-\Gamma_{\alpha m}^{i} \Gamma_{k l}^{\alpha} \quad \alpha=0, \ldots, 1+n .
$$

From Corollary 1 and the well known general relation

$$
\Gamma_{\mu \nu}^{\alpha}=\frac{1}{2} g^{\alpha \beta}\left(\frac{\partial g_{\beta \mu}}{\partial \xi^{\nu}}+\frac{\partial g_{\beta \nu}}{\partial \xi^{\mu}}-\frac{\partial g_{\mu \nu}}{\partial \xi^{\beta}}\right)
$$


one can easily check that the connection $\Gamma$ induced from $g^{E}$ has a particularily simple structure, which we write as

$$
\Gamma=\Gamma^{(1)} \oplus \Gamma^{(n)}
$$

with $\Gamma^{(1)}=\Gamma_{00}^{0}$ and $\Gamma^{(n)}=\left\{\Gamma_{j k}^{i}\right\}, i, j, k=1, \ldots, n$ corresponding to the metrics on $\gamma_{O^{\prime}}$ and $\Sigma_{O^{\prime}}$ respectively. Exploiting this fact, eq. (2.5) becomes

$$
R_{k l m}^{(1+n) i}=R_{k l m}^{(n) i}+\Gamma_{0 l}^{i} \Gamma_{k m}^{0}-\Gamma_{0 m}^{i} \Gamma_{k l}^{0}=R_{k l m}^{(n) i}
$$

The left hand side is zero by flatness, so that necessarily $R_{k l m}^{(n) i}=0$.

It is also worth to stress that, as usually done in the theory of Riemannian manifolds, we can introduce normal coordinates $\xi_{*}^{0}, \xi_{*}^{1}, \ldots, \xi_{*}^{n}$ in a neighborhood $I_{x}$ of each point $x \in \mathrm{R}^{1+n}$. This is obtained by choosing normal coordinates both in the one-dimensional neighborhood of the projection of $x$ on $\gamma_{O^{\prime}}$ and in the $n$-dimensional neighborhood of the projection of $x$ on $\Sigma_{O^{\prime}}$ along $C_{V}$. (Indeed, due to the flatness of $\Sigma_{O^{\prime}}$ (Corollary 3), this can be done globally, so that it possible to define a coordinate system that yields an orthonormal coordinate basis at every point of $\left.\mathrm{R}^{1+n}\right)$. Hence, in case $O^{\prime}$ coincides with $O$, the linear part of the transformation $\Phi_{*}: y \mapsto \xi_{*}:=\Phi_{*}(y)$ can be thought as a rotation on $\mathrm{R}^{1+n}$ which aligns the $x^{0}$-axis along the direction of the vector field and which is different from point to point if the latter is not constant.

\section{GLOBAL COMOVING COORDINATES IN MINKOWSKIAN SPACES AND PSEUDO-RIEMANNIAN MANIFOLDS}

In this section the Minkowski spacetime $\mathrm{M}^{4}$ is understood to be endowed with the standard Euclidean topology. (By the way, in the literature the so-called "path topology" has also been considered [14, nevertheless it looks quite unsuitable in our setting). Then it is straigthforward to extend Theorem 1: 
Theorem 1': Let $\mathrm{M}^{4}$ be equipped with the Euclidean topology and let $\eta_{\mu \nu}:=$ $\operatorname{diag}(-1,1,1,1)$ the Minkowskian metric tensor. If $V$ is a vector field on $\mathrm{M}^{4}$ with covariant components $\left(V_{0}, V_{1}, V_{2}, V_{3}\right)$ satisfying the following conditions:

i) $V_{0}(x) \neq 0 \quad \forall x \in \mathrm{M}^{4}$,

ii) There exists a scalar field $S \in \mathcal{C}^{r}\left(\mathrm{M}^{4}, \mathrm{R}\right), r \geq 2$, such that

$$
V_{\mu}(x)=\frac{\partial}{\partial x^{\mu}} S(x), \quad \forall \mu=0, \ldots, 3, \forall x \in \mathrm{M}^{4}
$$

then there exists a diffeomorphism $\Phi$ such that

$$
V^{\prime \mu}(x):=\sum_{\nu=0}^{n} \frac{\partial \Phi^{\mu}(x)}{\partial x^{\nu}} V^{\nu}(x)=\left\{\begin{array}{cl}
\sqrt{\left|V_{\mu}(x) V^{\mu}(x)\right|} & \text { if } \mu=0 \\
0 & \text { otherwise }
\end{array}\right.
$$

Proof: With the given topology, $\mathrm{M}^{4}$ is a pseudo-Riemannian manifold with a unique chart $\mathrm{R}^{4}$, cartesian natural coordinates and metric tensor $\eta_{\mu \nu}$. Thus the proof of Theorem 1 remains the same as far as it does not involve the metric tensor. This occurs only where we assert that the vector $V(x)$ is orthogonal to $\Sigma_{x}$ at point $x$. But we have seen that the Euclidean product between a vector with (covariant) components $\partial S(x) / \partial x^{\mu}$ and any vector $\hat{e}(x) \in \mathrm{R}^{4}$ which is tangent to $\Sigma_{O^{\prime}}$ at point $x$ is equal to zero. Hence we can write

$$
\frac{\partial}{\partial x^{\mu}} S(x) \hat{e}^{\mu}(x)=V_{\mu}(x) \hat{e}^{\mu}(x)=\eta_{\mu \nu} V^{\mu} \hat{e}^{\nu}(x)=0
$$

so that, by the arbitrariness of the tangent vector $\hat{e}(x)$, the vector $V(x)=\left(V^{0}, V^{1}, V^{2}, V^{3}\right)$ is orthogonal to $\Sigma_{x}$ at the point $x$ in the Minkowskian metric.

We shall now for definiteness suppose that the congruence $C_{V}$ is timelike. Corollaries 1 , 2 and 3 can be easily extended in the following way.

Corollary $1^{\prime}$ : Let $\xi^{0}=\lambda$ be any coordinate on $\left(O^{\prime}, \lambda\right)$. Then introducing on $\gamma_{O^{\prime}}$ the negative metric function $g_{00}(\lambda)$ we have

$$
V^{\prime \mu}(x)=\left\{\begin{array}{cl}
\frac{\sqrt{-V^{\mu}(x) V_{\mu}(x)}}{\sqrt{-g_{00}(\lambda(x))}} & \text { if } \mu=0 \\
0 & \text { otherwise }
\end{array}\right.
$$


Corollary 2': The global coordinate transformation $\Phi: x \mapsto \xi:=\Phi(x), x \in \mathrm{M}^{4}$ transforms the Minkowskian metric tensor $\eta_{\mu \nu}$ into the tensor $g_{\mu \nu}$ defined by

$$
g_{\mu \nu}(\xi)=\left\{\begin{array}{cl}
g_{00}\left(\xi^{0}\right) & \mu=\nu=0, \\
0 & \mu=0, \nu=1,2,3 ; \nu=0, \mu=1,2,3, \\
\sigma_{\mu \nu}\left(\xi^{1}, \xi^{2}, \xi^{3}\right) & \text { otherwise } .
\end{array}\right.
$$

Proof: This structure immediately comes from that of $g^{E}$. In fact we can write

$$
g_{\mu \nu}^{E}(\xi)=\delta_{\alpha \beta} \frac{\partial \Phi^{-1 \alpha}}{\partial \xi^{\mu}}(\xi) \frac{\partial \Phi^{-1 \beta}}{\partial \xi^{\nu}}(\xi)
$$

and

$$
g_{\mu \nu}(\xi)=\eta_{\alpha \beta} \frac{\partial \Phi^{-1 \alpha}}{\partial \xi^{\mu}}(\xi) \frac{\partial \Phi^{-1 \beta}}{\partial \xi^{\nu}}(\xi)
$$

so that a comparison between (3.1) and (3.2) yields $g_{\mu \nu}=g_{\mu \nu}^{E}$ for $\mu \neq 0$ and $\nu \neq 0$ while $g_{00}\left(\xi^{0}\right)=-g_{00}^{E}\left(\xi^{0}\right)=-f\left(\xi^{0}\right)$.

Corollary 3 still holds (the same proof with $n=3$ ).

Exactly as before, it is possible to introduce normal coordinates $\xi_{*}$ in a neighborhood $I_{x}$ of any point $x \in \mathrm{M}^{4}$ by projecting $I_{x}$ on $\Sigma_{O^{\prime}}$ along $C_{V}$. In this case, if $O^{\prime}$ coincides with $O$, the coordinate transformation $\Phi_{*}: y \mapsto \xi_{*}:=\Phi_{*}(y), y \in I_{x}$ has the linear part which reduces, up to a spatial rotation, to a Lorentz boost associated to $V(x)$, or, explicitely:

$$
\left(\frac{\partial \Phi_{*}^{\mu}}{\partial x^{\nu}}\right)(x)=\Lambda_{\nu}^{-1 \mu}(x) \quad \forall x \in \mathrm{M}^{4} .
$$

Theorem $1^{\prime}$ can also be generalized to vector fields defined on a pseudo-Riemannian manifold $\mathrm{M}$. In fact, given a vector field $\hat{V}$ on $\mathrm{M}$, let $x=\left(x^{0}, x^{1}, \ldots, x^{n}\right)$ denote the local coordinates of a point $\mathrm{p} \in \mathrm{M}$ and $\left(V_{0}(x), V_{1}(x), \ldots, V_{n}(x)\right)$ the covariant components of $\hat{V}$ at the same point. Thus, in case for any point $\mathrm{p} \in \mathrm{M}$ one has $V_{0}(x) \neq 0$ and $V_{\mu}(x)=\partial S(x) / \partial x^{\mu}$ for some $S \in \mathcal{C}^{r}\left(U_{\mathrm{p}}, \mathrm{R}\right), r \geq 2$ and $U_{\mathrm{p}}$ denoting the local chart, we can apply Theorem $1^{\prime}$ 
(or the analogous for a metric having a different signature) to get the desired coordinate transformation on the generic chart $U_{\mathrm{p}}$.

A local version of this global result is commonly quoted in General Relativity textbooks.

\section{STOCHASTIC QUANTIZATION OF THE FREE RELATIVISTIC SPINLESS PARTICLE}

Nelson's stochastic kinematics for a spinless quantum particle is obtained by promoting the classical configuration variables to a Markov diffusion with constant coefficient equal to $\hbar / m, \hbar$ being reduced Planck's constant and $m$ the mass of the particle:

$$
d q(t)=b(q(t), t) d t+\left(\frac{\hbar}{m}\right)^{\frac{1}{2}} d w(t), \quad t \in[0,+\infty)
$$

As briefly exposed in Sec. I, the hydrodynamical (or Eulerian) picture of the diffusion process is given, under appropriate regularity conditions, in terms of the time dependent density $\rho$ and the current velocity $v$. The dynamics is then assigned by imposing that the motion extremizes the mean (regularized) classical action written as a functional of both fields [10], [15]. In the case of a particle subjected to a scalar potential $\mathcal{V}$, the time evolution of $\rho$ and $v$ is described by two coupled partial differential equations:

$$
\left\{\begin{array}{l}
\partial_{t} \rho+\nabla \cdot(\rho v)=0 \\
\partial_{t} v+(v \cdot \nabla) v+\frac{\hbar^{2}}{2 m^{2}} \nabla\left(\frac{\nabla^{2} \sqrt{\rho}}{\sqrt{\rho}}\right)=-\nabla \mathcal{V},
\end{array}\right.
$$

in which $v$ must be irrotational in all points where $\rho$ is positive. Introducing therefore a scalar field $S$ such that $v=\nabla S / m$ and performing the standard substitution

$$
\psi(x, t):=\sqrt{\rho(x, t)} \exp \left\{\frac{i}{\hbar} S(x, t)\right\}
$$

one can transform (4.2) into the Schrödinger wave-equation

$$
i \hbar \frac{\partial \psi}{\partial t}=\left[-\frac{\hbar^{2}}{2 m} \nabla^{2}+m \mathcal{V}\right] \psi
$$

Our objective is now to extend such a procedure to the quantization of a free relativistic spinless particle. This will be achieved by exploiting a suitable comoving coordinate system 
and starting, as previously done [12], from the classical Einstein action. We conjecture that the quantum evolution equation can be written also in the relativistic situation in terms of a couple of fields $(\rho, v)$; they are expected to satisfy a covariant hydrodynamic equation which reduces to (4.2) (for $\mathcal{V}=0$ ) in the limit $\|v\| \ll c$. The relevant comoving coordinate system will be that induced by the four-velocity field $V$ associated to $v$. Notice that the field $V$ enters at this level as an unknown; the fact that it fulfills both assumptions of Theorem $1^{\prime}$ will be verified a posteriori. To assign the kinematics we also need to introduce an evolution parameter independent of the particular choice of the coordinate $\lambda$ on $\gamma_{O^{\prime}}$. To this end let as before $l(\lambda)$ denote the length of the arc $\left(O^{\prime}, \lambda\right)$ of $\gamma_{O^{\prime}}$ and consider a temporal parameter $t$ defined by $t:=l(\lambda) / c$. By construction we have

$$
d l(\lambda)^{2}=d \xi^{0} d \xi_{0}=-f(\lambda) d \lambda^{2}=-c^{2} d t^{2} \quad, \quad d t=\frac{\sqrt{-d l(\lambda)^{2}}}{c}=\frac{1}{c} \sqrt{-g_{00}\left(\xi^{0}\right)} d \xi^{0}
$$

The physical meaning of this choice is that $t$ coincides with the proper time of the comoving observer having $\gamma_{O^{\prime}}$ as its world-line. In the following we will also identify $O=O^{\prime}$.

We formulate the stochastic kinematics of a free relativistic spinless particle as follows:

$\left.h_{1}\right) \quad$ there exists a timelike four-velocity field $V$ on $\mathrm{M}^{4}$, satisfying the assumptions of Theorem 1', such that the evolution of the position of the particle with respect to the parameter $t$ is described by a Markov diffusion in the comoving coordinate system associated to $V$; the diffusion coefficient is equal to $\hbar / m, m$ being the rest mass of the particle;

$h_{2}$ ) the current velocity of such a diffusion is equal to zero.

Denoting by $q^{i}, i=1,2,3$, the spatial comoving coordinates of the particle on $\Sigma_{0}$, the time development of the Markov process is fully specified by writing

$$
d q^{i}(t)=\beta_{+}^{i}(q(t), t) d t+\left(\frac{\hbar}{m}\right)^{\frac{1}{2}} G_{k}^{i}(q(t)) d w^{k}(t)
$$

where $w$ is a standard Wiener process on $\mathrm{R}^{3}$ and $G_{k}^{i} G^{k j}=g^{i j}, g^{i j}=\sigma^{i j}$ being the components of the (positive-definite) metric tensor of $\Sigma_{O}$ (Corollary $2^{\prime}$ ). We will also assume that 
the functions $\beta_{+}^{i}$ are sufficiently smooth to allow the existence of the so-called "backward representation" of the process [10,11], namely

$$
d q^{i}(t)=\beta_{-}^{i}(q(t), t) d t+\left(\frac{\hbar}{m}\right)^{\frac{1}{2}} G_{k}^{i}(q(t)) d w_{*}^{k}(t)
$$

where $w_{*}$ is a reversed standard Wiener process.

By introducing the infinitesimal forward and "backward" increments

$$
\begin{aligned}
& d^{+} q^{i}(t):=q^{i}(t+d t)-q^{i}(t) \\
& d^{-} q^{i}(t):=q^{i}(t)-q^{i}(t-d t)
\end{aligned}
$$

we can construct the two four-vectors

$$
\begin{aligned}
& d^{+} \xi^{\mu}:=\left(d \lambda, d^{+} q^{i}(t)\right) \\
& d^{-} \xi^{\mu}:=\left(d \lambda, d^{-} q^{i}(t)\right)
\end{aligned}
$$

in terms of which we define the mean regularized invariant

$$
\begin{aligned}
E\left\{d^{+} \xi^{\mu} d^{-} \xi_{\mu}\right\} & =E\left\{d^{+} q^{i}(t) d^{-} q_{i}(t)\right\}-c^{2} d t^{2} \\
& =E\left\{v_{+}^{i}(q(t)) v_{-i}(q(t))\right\} d t^{2}-c^{2} d t^{2}
\end{aligned}
$$

Here eq. (4.4) has been used and $v_{+}, v_{-}$are to so-called "invariant drifts" introduced in Refs. [10] and [16]; they are vector fields on $\Sigma_{O}$ defined by

$$
\begin{aligned}
& v_{+}^{i}:=\beta_{+}^{i}+\frac{\hbar}{2 m} g^{j k} \Gamma_{j k}^{i} \\
& v_{-}^{i}:=\beta_{-}^{i}-\frac{\hbar}{2 m} g^{j k} \Gamma_{j k}^{i},
\end{aligned}
$$

$\Gamma_{j k}^{i}$ being the Christoffel symbols associated to $g_{i j}$ (metric connection).

As usual in Stochastic Mechanics, we introduce the current velocity $\beta$ and the osmotic velocity $u$ by combining the drifts:

$$
\begin{aligned}
& \beta:=\frac{1}{2}\left(v_{+}+v_{-}\right)=\frac{1}{2}\left(\beta_{+}+\beta_{-}\right) \\
& u:=\frac{1}{2}\left(v_{+}-v_{-}\right) .
\end{aligned}
$$

It is convenient to introduce a smooth invariant measure on the manifold $\Sigma_{O}$ and define the relative covariant probability density of the process, $\rho(q, t)$, through the equality 


$$
E\{F(q(t))\}:=\int_{\mathrm{R}^{3}} F(q) \rho(q, t) \sqrt{|g|} d^{3} q, \quad|g|:=\operatorname{det}\left[g_{i j}\right]
$$

where $F(q)$ is any Lebesgue integrable function of the process. It is well known [10] that, in case $\rho$ is strictly positive, $\beta, u$ and $\rho$ are connected by the following relations:

$$
\begin{gathered}
u^{i}=\frac{\hbar}{2 m} \nabla^{i} \ln \rho \\
\partial_{t} \rho+\nabla_{i}\left(\rho \beta^{i}\right)=0,
\end{gathered}
$$

where $\nabla_{i}$ denotes the covariant derivative along direction $q_{i}$ (recall that by construction $\left.\nabla_{i} g_{j k}=0 \forall i, j, k\right)$. For the extension to the case when $\rho$ has zeroes see [11]. According to $h_{2}$ ), we should also put $\beta$ equal to zero (so that $\beta_{ \pm}$become time independent); we prefer to do this later, in order to show that this choice is naturally enforced by consistency requirements.

Thus, in analogy with the classical case, we consider, for arbitrary events $a$ and $b$ in $\mathrm{M}^{4}$, the following stochastic version of Einstein's action:

$$
A_{[a, b]}:=-m c \int_{a}^{b} \sqrt{E\left\{-d^{+} \xi^{\mu} d^{-} \xi_{\mu}\right\}}=-m c^{2} \int_{t_{a}}^{t_{b}} \sqrt{1-E\left\{\frac{v_{+}^{i} v_{-i}}{c^{2}}\right\}} d t
$$

which can be written as a functional of $\beta$ and $\rho$ exploiting (4.10) and (4.11a). We get

$$
A_{[a, b]}=-m c^{2} \int_{t_{a}}^{t_{b}} d t\left(1-\frac{1}{c^{2}} \int_{\mathrm{R}^{3}}\left(\beta^{2}-u^{2}\right) \rho \sqrt{|g|} d^{3} q\right)^{\frac{1}{2}}
$$

with the convention $\beta^{2}:=\beta^{i} \beta_{i}=g_{i j} \beta^{i} \beta^{j}$ and similarly for $u^{2}$. We recall here that, since the configurational manifold has zero intrinsic curvature, no Pauli-DeWitt curvature term 17 has to be considered in the expression of the stochastic action functional.

Following a variational strategy which is analogous to that exploited in non relativistic Stochastic Mechanics [15], we can now extremize the action with respect to independent variations $\delta \beta$ and $\delta \rho$. Since $\rho$ and $\beta$ are linked by the continuity equation (4.11b), this may be done by introducing a Lagrangian multiplier $\chi$, so that we construct the modified action

$$
\bar{A}_{[a, b]}:=A_{[a, b]}+\int_{t_{a}}^{t_{b}} d t \int_{\mathrm{R}^{3}} \chi\left[\partial_{t} \rho+\nabla_{i}\left(\rho \beta^{i}\right)\right] \sqrt{|g|} d^{3} q
$$

We require that 


$$
\begin{aligned}
\delta_{v} \bar{A}_{[a, b]} & =o(\delta \beta) \\
\delta_{\rho} \bar{A}_{[a, b]} & =o(\delta \rho)
\end{aligned}
$$

for independent variations $\delta \beta$ and $\delta \rho$ having compact support on $\Sigma_{0} \times\left[t_{a}, t_{b}\right]$. A straigthforward calculation yields the two variational equations

$$
\begin{gathered}
\frac{m \beta_{k}}{\sqrt{1-E\left\{\frac{\beta^{2}}{c^{2}}-\frac{u^{2}}{c^{2}}\right\}}}=\nabla_{k} \chi, \quad k=1,2,3, \\
\frac{1}{2} \frac{m \beta^{2}}{\sqrt{1-E\left\{\frac{\beta^{2}}{c^{2}}-\frac{u^{2}}{c^{2}}\right\}}}+\frac{m}{\sqrt{1-E\left\{\frac{\beta^{2}}{c^{2}}-\frac{u^{2}}{c^{2}}\right\}}}\left\{\frac{1}{2} u^{2}+\frac{\hbar}{2 m} \nabla_{i} u^{i}\right\}-\frac{\partial \chi}{\partial t}-\nabla_{i} \chi \beta^{i}=0 .
\end{gathered}
$$

We eliminate $\chi$ by taking the gradient of the second equation; we are left with the following three-dimensional dynamical equation:

$$
\begin{aligned}
\frac{\partial}{\partial t} \frac{\beta_{k}}{\sqrt{1-E\left\{\frac{\beta^{2}}{c^{2}}-\frac{u^{2}}{c^{2}}\right\}}}+ & \left(\beta_{i} \nabla^{i}\right) \frac{\beta_{k}}{\sqrt{1-E\left\{\frac{\beta^{2}}{c^{2}}-\frac{u^{2}}{c^{2}}\right\}}} \\
& -\left[\frac{\hbar}{2 m} \frac{\nabla_{i} \nabla^{i} u_{k}}{\sqrt{1-E\left\{\frac{\beta^{2}}{c^{2}}-\frac{u^{2}}{c^{2}}\right\}}}+\left(u_{i} \nabla^{i}\right) \frac{u_{k}}{\sqrt{1-E\left\{\frac{\beta^{2}}{c^{2}}-\frac{u^{2}}{c^{2}}\right\}}}\right]=0,
\end{aligned}
$$

being $\nabla_{i} \nabla^{i}$ the ordinary three-dimensional Laplace-Beltrami operator.

In order to get a covariant expression of (4.15), we need a fourth equation which in the classical case is obtained by imposing that the action be stationary with respect to variations of the evolution parameter and which represents the conservation of energy. In this way the Hamiltonian is constructed as the conjugate momentum with respect to time. Let us then consider $t$ as a new dynamical variable $t=t(\tau)$, function of some invariant auxiliary parameter $\tau$. Since the Lagrangian does not depend explicitly on $t$, the requirement of stationarity of (4.12) with respect to smooth variations of $t$ having compact support in $\left[\tau_{a}, \tau_{b}\right]$, that is

$$
\delta_{t} A_{\left[t\left(\tau_{a}\right), t\left(\tau_{b}\right)\right]}=o(\delta t)
$$


immediately yields

$$
\frac{d}{d t} \frac{m c^{2}}{\sqrt{1-E\left\{\frac{\beta^{2}}{c^{2}}-\frac{u^{2}}{c^{2}}\right\}}}=0
$$

Alternatively we can also identify the Hamiltonian with a first integral of the motion. If $\left(\rho_{*}, \beta_{*}\right)$ denotes a given solution of (4.15) and (4.11b), then in the total variation of $\bar{A}$ only the boundary term survive:

$$
\delta_{\rho_{*}, \beta_{*}} \bar{A}_{[a, b]}=\int_{t_{a}}^{t_{b}} d t \frac{\partial}{\partial t} \int_{\mathrm{R}^{3}} \chi \delta \rho_{*} \sqrt{|g|} d^{3} q
$$

We particularize now the variations in the following way:

$$
\begin{aligned}
& \delta \rho_{*}=\dot{\rho}_{*} \delta t+o(\delta t) \\
& \delta \beta_{*}=\dot{\beta}_{*} \delta t+o(\delta t),
\end{aligned}
$$

so that expression (4.17) becomes

$$
\delta_{\rho_{*}, \beta_{*}} \bar{A}_{[a, b]}=\delta t \cdot \int_{t_{a}}^{t_{b}} d t \frac{\partial}{\partial t} \int_{\mathrm{R}^{3}}\left(\chi \dot{\rho}_{*}\right) \sqrt{|g|} d^{3} q+o(\delta t)
$$

On the other hand, being the dynamics time independent one can also write

$$
\delta_{\rho_{*}, \beta_{*}} \bar{A}_{[a, b]}=\delta t \cdot \int_{t_{a}}^{t_{b}} d t\left(\frac{d}{d t} \bar{L}\right)_{\rho_{*}, \beta_{*}}+o(\delta t)
$$

so that, comparing (4.18) and (4.19) and taking the limit for $\delta t$ going to zero, we find

$$
\frac{d}{d t}\left[\int_{\mathrm{R}^{3}}(\chi \dot{\rho}) \sqrt{|g|} d^{3} q-m c^{2} \sqrt{1-E\left\{\frac{\beta^{2}}{c^{2}}-\frac{u^{2}}{c^{2}}\right\}}\right]=0 .
$$

for any solution $(\rho, \beta)$ of $(4.15)$ and (4.11b) (we note that $\bar{L}=L$ in this case). From this, continuity equation and an integration by parts give

$$
\frac{d}{d t} \frac{m c^{2}+E\left\{m u^{2}\right\}}{\sqrt{1-E\left\{\frac{\beta^{2}}{c^{2}}-\frac{u^{2}}{c^{2}}\right\}}}=0 .
$$

As a consequence, we can verify that a natural assumption in order the energy be properly defined is that the current velocity $\beta$ in the comoving coordinates be equal to zero. 
Under this assumption, the density of the process is made time independent so that $d E\left\{u^{2}\right\} / d t=0$ by (4.11a) and (4.21) becomes identical to (4.16). This discussion shows that hypothesis $h_{2}$ ) on the kinematics is in fact crucial in formulating a mathematically and physically consistent theory.

Putting explicitely hereafter $\beta=0$ and defining the constant functional

$$
\tilde{\gamma}:=\left(1+E\left\{\frac{u^{2}}{c^{2}}\right\}\right)^{-\frac{1}{2}}=\tilde{\gamma}[\rho],
$$

we get the following equations of motion in the comoving coordinates:

$$
\left\{\begin{array}{l}
\frac{\hbar \tilde{\gamma}}{2 m} \nabla_{i} \nabla^{i} u_{k}+\left(u_{i} \nabla^{i}\right) \tilde{\gamma} u_{k}=0 \\
\frac{d}{d t} \tilde{\gamma} m c^{2}=0 .
\end{array}\right.
$$

It is apparent that for every fixed solution $u$ of (4.23) the quantity $\tilde{\gamma}$ acts as a strictly positive constant; in fact, it drops out from the equations and we can solve the equivalent problem given by

$$
\left\{\begin{array}{l}
\frac{\hbar^{2}}{2 m} \nabla_{i} \nabla^{i} u_{k}+\hbar\left(u_{i} \nabla^{i}\right) u_{k}=0 \\
\frac{\partial}{\partial \xi^{0}} m c^{2}=0
\end{array}\right.
$$

where the original comoving coordinates $\left(\xi^{0}, \xi^{i}\right)$ are reintroduced (recall that $\nabla_{i}=\nabla_{q^{i}}=$ $\left.\nabla_{\xi^{i}}, i=1,2,3\right)$.

In order to express (4.24) in a covariant form, one has to introduce objects with the correct transformation properties. First of all, let us define a real field $\tilde{p}$ by

$$
\tilde{p}(\xi):=\mathcal{N} \rho(q), \quad \mathcal{N}=\left(\int_{-\Delta}^{\Delta} \sqrt{-g_{00}\left(\xi^{0}\right)} d \xi^{0}\right)^{-1},
$$

where the constant $\mathcal{N}$ has been chosen by demanding that $\tilde{p}(\xi)$ is a normalized probability density on a fixed four-dimensional domain $\Omega:=[-\Delta, \Delta] \times \mathrm{R}^{3}, \Delta \in \mathrm{R}^{+}$:

$$
\int_{\Omega} \tilde{p}(\xi) \sqrt{\left|g_{\mu \nu}\right|} d^{4} \xi=\int_{-\Delta}^{\Delta} \mathcal{N} \sqrt{-g_{00}\left(\xi^{0}\right)} d \xi^{0} \int_{\mathrm{R}^{3}} \sqrt{|g|} d^{3} q \rho(q)=1,
$$

with $\mid g_{\mu \nu \mid}:=\operatorname{det}\left[g_{\mu \nu}\right]$. By construction $\tilde{p}$ is a density with respect to the invariant measure on $\Omega$, so that it transforms as a scalar. 
As a second step, we introduce also the two four-dimensional fields with components

$$
\tilde{V}^{\mu}=\left(\frac{c}{\sqrt{-g_{00}\left(\xi^{0}\right)}}, 0\right) \quad, \quad \tilde{U}^{\mu}=\left(0, u^{i}\right) .
$$

Both $\tilde{U}$ and $\tilde{V}$ are four-vectors, due to different reasons. For the latter the four-vector character directly follows from the tensorial relation:

$$
\tilde{U}^{\mu}=\frac{\hbar}{2 m} \nabla^{\mu} \ln \tilde{p}
$$

which we obtain from (4.11a) and (4.25). The former is instead the four-velocity field corresponding to the current velocity vector $\beta$ written in comoving coordinates (where the spatial part $\beta$ is identically vanishing). It can be noticed that $\tilde{V}^{\mu}$ satisfy itself a relation similar to (4.28):

$$
\tilde{V}^{\mu}=: \frac{1}{m} \nabla^{\mu} \tilde{S} \quad, \quad \tilde{S}(\xi):=-m c \int_{-\infty}^{\xi^{0}} d \bar{\xi}^{0} \sqrt{-g_{00}\left(\bar{\xi}^{0}\right)} .
$$

It is important to recognize that eq. (4.29) automatically defines $\tilde{S}$ as a scalar field. In all generality, the metric function $g_{00}\left(\xi^{0}\right)$ must be negative definite, normalized according to (4.26) and sufficiently smooth to guarantee the existence of the primitive in (4.29). The simplest choice is obviously to take $g_{00}\left(\xi^{0}\right)=-1$, or, physically, to adopt the proper time of the comoving observer along $\gamma_{O}$ as a coordinate, $d \xi^{0}:=c d t$. In this case, the function $\tilde{S}$ simply reduces to

$$
\tilde{S}(\xi)=-m c \xi^{0} \quad, \quad \xi^{0}=c t
$$

Maintaining the above made positions and exploiting the fact that, by (4.27), $\tilde{U}^{0}=0$, $\tilde{V}^{i}=0, i=1,2,3$, we can now easily rewrite eqs. (4.24) as follows

$$
g^{\alpha \beta}\left[-\left(\frac{\hbar^{2}}{2 m} \nabla_{\alpha} \nabla_{\beta} \tilde{U}^{\mu}+\hbar \tilde{U}_{\alpha} \nabla_{\beta} \tilde{U}^{\mu}\right)+\hbar \tilde{V}_{\alpha} \nabla_{\beta} \tilde{V}^{\mu}\right]=0 .
$$

The continuity equation can also be rewritten, in terms of a four-dimensional divergence:

$$
\nabla_{\mu}\left[\tilde{p} \tilde{V}^{\mu}\right]=0
$$


Now, by means of the same change of variables exploited to get the Schrödinger equation (4.3) from its hydrodynamical counterpart (4.2), it can be easily realized that (4.31) and (4.32) correspond respectively to the real and imaginary part of a stationary four-dimensional Schrödinger equation, namely

$$
-\frac{\hbar^{2}}{2 m} g^{\alpha \beta} \nabla_{\alpha} \nabla_{\beta} \tilde{\phi}=\mu \tilde{\phi}
$$

where

$$
\tilde{\phi}(\xi):=\sqrt{\tilde{p}(\xi)} \exp \left\{\frac{i}{\hbar} \tilde{S}(\xi)\right\}
$$

We remark at this point that, had we maintained $\tilde{\gamma}$ (eq. (4.22)) in the derivation, then we would have found an apparently non linear equation, which is quoted in [12]. We observe also that, as usual in Stochastic Mechanics, the eigenvalue $\mu$ is equal to the mean value of an energy. In the particular case one can show by direct computation that

$$
\mu=\int_{\mathrm{R}^{3} \times[-\Delta, \Delta]}\left\{\frac{1}{2} m \tilde{V}_{\mu} \tilde{V}^{\mu}+\frac{1}{2} m \tilde{U}_{\mu} \tilde{U}^{\mu}\right\} \tilde{p}(\xi) \sqrt{\left|g_{\mu \nu}\right|} d^{4} \xi=-\frac{1}{2} m c^{2}+\frac{1}{2} m E\left\{u^{2}\right\} .
$$

We notice that, since $u$ depends on the quantum state $\tilde{\phi}$ through $\tilde{p}$, the Schrödinger-like equation (4.33) exhibits a spectrum of energy levels. On the other hand, one can also observe that the ratio of the two contributions in (4.35) is

$$
\frac{E\left\{u^{2}\right\}}{c^{2}}=\frac{\hbar^{2}}{4 m^{2} c^{2}} \int_{\mathrm{R}^{3}} \nabla_{\mu} \ln \rho \nabla^{\mu} \ln \rho \sqrt{|g|} d^{3} q,
$$

so that, avoiding extremely sharp densities, which would be patological in the free case, one can put with good approximation

$$
E\left\{u^{2}\right\} / c^{2} \ll 1
$$

This approximation acquires a clear physical significance if the right hand side of (4.36) is interpreted as the (squared) ratio between the Compton wavelength $\hbar / m c$ and the typical length scale associated to the density $\rho$. Converting to energy units, one finds that the typical energy involved in the description has to be much less than $2 m c^{2}$. This is precisely 
the energy threshold under which a relativistic single particle theory is expected to hold. Turning to the four-dimensional Schrödinger equation (4.33), validity of (4.37) means that energy levels become degenerate and consequently the spectrum collapses to

$$
\mu \approx-\frac{1}{2} m c^{2}
$$

Incidentally, this relation, also called "Feynman ansatz", represents a widely accepted working assumption in the literature [3], [18]. Equation (4.33) reduces then to the Klein-Gordon equation in comoving coordinates:

$$
\left[g^{\alpha \beta} \nabla_{\alpha} \nabla_{\beta}-\frac{m^{2} c^{2}}{\hbar^{2}}\right] \tilde{\phi}(\xi)=0, \quad \xi \in \Omega .
$$

Transforming to cartesian coordinates $\left\{x^{\mu}\right\}$ on $\mathrm{M}^{4}$, we find from this the familiar expression

$$
\left[\eta^{\mu \nu} \frac{\partial}{\partial x^{\mu}} \frac{\partial}{\partial x^{\nu}}-\frac{m^{2} c^{2}}{\hbar^{2}}\right] \phi(x)=0, \quad x \in \Phi^{-1}(\Omega),
$$

where, from (4.34),

$$
\phi(x):=\sqrt{p(x)} \exp \left\{\frac{i}{\hbar} S(x)\right\}=\sqrt{\tilde{p}(\xi(x))} \exp \left\{\frac{i}{\hbar} \tilde{S}((\xi(x))\} .\right.
$$

Since the parameter $\Delta$ which fixes the domain $\Omega$ is arbitrary, the region where the equation is defined can be sufficiently large to contain any arbitrarily chosen rectangle in $\mathrm{M}^{4}$.

\section{STOCHASTIC INTERPRETATION OF DIFFERENT SUBSETS OF SOLUTIONS TO KLEIN-GORDON EQUATION AND NON RELATIVISTIC LIMIT}

The quantization procedure which we have just outlined naturally selects a particular subset of the solutions to Klein-Gordon equation. In fact we observe that, in comoving coordinates, the conserved four-current density at an arbitrary point $x \in \mathrm{M}^{4}$, defined as

$$
\tilde{J}^{\mu}(\xi):=m \tilde{p}(\xi) \tilde{V}^{\mu}(\xi)=\tilde{p}(\xi) \nabla^{\mu} \tilde{S}(\xi), \quad \xi=\Phi(x)
$$

has the following particular structure: 


$$
\tilde{J}^{0}(\xi)=\frac{m c \tilde{p}(\xi)}{\sqrt{-g_{00}\left(\xi^{0}\right)}} \quad, \quad \tilde{J}^{i}(\xi)=0 .
$$

We also notice, that the conservation law associated to (5.2), namely

$$
\nabla_{\mu} \tilde{J}^{\mu}=0
$$

is nothing but a rewriting of the continuity equation (4.32) for the probability density of the stationary diffusion $q(t)$.

For a generic solution of the Klein-Gordon equation in the inertial frame let us correspondly introduce the conserved four-current density by

$$
J_{\mu}(x):=|\phi(x)|^{2} \frac{\partial}{\partial x^{\mu}} S(x)=\frac{\hbar}{2 i}\left(\phi^{*} \partial_{\mu} \phi-\phi \partial_{\mu} \phi^{*}\right) .
$$

Denoting by $V(x)$ the four-vector with covariant components $V_{\mu}(x)=\partial_{\mu} S(x) / m$, we have by construction

$$
\tilde{V}^{\mu}(\xi)=\frac{\partial \Phi^{\mu}}{\partial x^{\nu}}(x) V^{\nu}(x), \quad \xi=\Phi(x) .
$$

We then choose in a neighborhood of $\xi$ normal coordinates $\xi_{*}:=\left(\xi_{*}^{0}, \xi_{*}^{1}, \xi_{*}^{2}, \xi_{*}^{3}\right)$. As observed in Sec. III, we get

$$
\tilde{V}_{*}^{\mu}(\xi)=\Lambda_{\nu}^{-1 \mu}(x) V^{\nu}(x),
$$

where $\tilde{V}_{*}^{\mu}$ denote the normal components of $\tilde{V}$ and $\Lambda_{\nu}^{\mu}$ is, up to a spatial rotation, the Lorentz boost associated to the three-dimensional velocity

$$
v^{i}:=c \frac{V^{i}}{V^{0}}, \quad i=1,2,3 .
$$

Thus we can conclude that the comoving coordinate transformation $\Phi$, represented in normal coordinates in the neighborhood of a point $x$ by $\Phi=\Phi_{*}$, acts on four-vectors as a Lorentz boost associated to the three-velocity $v(x)$ (which is uniquely determined by the four-velocity $V(x))$.

We also observe that since $J^{\mu}$ is itself a four-vector, then 


$$
J^{\mu}(x)=\Lambda_{0}^{\mu}(\xi) \tilde{J}_{*}^{0}(\xi)
$$

with (from (5.2))

$$
\tilde{J}_{*}^{0}(\xi)=m c \tilde{p}(\xi)
$$

Recalling the transformation properties of $\tilde{p}$ and expliciting the Lorentz boost, one finds from $(5.7)-(5.8)$

$$
J^{0}=\frac{m c p}{\sqrt{1-\frac{v^{2}}{c^{2}}}} \quad, \quad J^{i}=\frac{m v^{i} p}{\sqrt{1-\frac{v^{2}}{c^{2}}}}
$$

so that

$$
\left\{\begin{array}{l}
J^{0} \geq 0 \\
J_{\mu} J^{\mu}=-m^{2} c^{2}|\phi|^{2} .
\end{array} \quad \forall x \in \Phi^{-1}(\Omega) .\right.
$$

Thus we can immediately recognize that a necessary and sufficient condition for a solution to Klein-Gordon equation corresponds to a one-particle diffusion satisfying the variational principle in the comoving coordinates is that conditions (5.10) hold.

In fact, necessity was just proven. Conversely, given a normalized solution of the KleinGordon equation with associated four-current density $J$, sufficiency immediately comes by putting, in all points where $J^{0} \neq 0$,

$$
v^{i}:=c \frac{J^{i}}{J^{0}} \quad, \quad p:=\phi^{*} \phi .
$$

We have from this

$$
J_{\mu} J^{\mu}=-\left(J^{0}\right)^{2}\left(1-\frac{v^{2}}{c^{2}}\right)=-m^{2} c^{2} p^{2}
$$

and consequently

$$
J^{0}=\frac{m c p}{\sqrt{1-\frac{v^{2}}{c^{2}}}}=\Lambda_{\mu}^{0} \tilde{J}_{*}^{\mu} \quad, \quad J^{i}=\frac{m v^{i} p}{\sqrt{1-\frac{v^{2}}{c^{2}}}}=\Lambda_{\mu}^{i} \tilde{J}_{*}^{\mu},
$$

which proves the assertion. 
Some remarks are now in order. First of all, we notice that the set of solutions restricted by conditions (5.10) is not empty since it contains at least all positive frequency plane-wave solutions (in a suitable limit of non normalized $\phi$ ). Note also that the positivity constraint on $J^{0}$ consistently allows to interpret it as a conserved density in hydrodynamical sense.

Furthermore, this stochastic quantization procedure can be extended in an obvious way to the description of a beam of identical (spinless) non interacting particles: in this case, one would simply recover positive energy solutions fulfilling both (5.10) but such that

$$
\int_{\mathrm{M}^{4}}|\phi|^{2} d^{4} x=N
$$

$N$ denoting the total number of particles. (This is trivially obtained by substituting the non normalized density $\rho N$ in place of $\rho$ at the beginning of our derivation).

A less obvious fact is that it is possible to give a fully probabilistic interpretation also to solutions with negative energy. The idea can be sketched as follows. Let us start from a $\mathrm{R}^{3}$-valued diffusion having zero current velocity and satisfying the stationary stochastic differential equation

$$
d q(t)=\beta_{+}(q(t)) d t+\nu^{\frac{1}{2}} d w(t)=u(q(t)) d t+\nu^{\frac{1}{2}} d w(t), \quad t \in[0, T], d t>0,
$$

with $u$ denoting, as usual, the osmotic velocity and $T>0$ arbitrary. Let us now think of a diffusion with a "specular" time evolution. In order to have an example of a (classical) phenomenon that would be described by means of such a "specular" diffusion, one could imagine a cloud of brownian particles which, starting from an initial spreaded spatial distribution, would concentrate after some interval of time in a small region. This is a "rare" event to which usual probabilistic models would give zero probability, but which is not, of course, in principle impossible. To get a mathematical description in terms of "forward" differentials, let us firstly introduce time-reversal in the usual way:

$$
\left\{\begin{array}{l}
t^{\prime}=-t \\
q^{\prime}\left(t^{\prime}\right)=q(t) \\
q^{\prime}\left(t^{\prime}-\delta\right)=q(t+\delta),
\end{array}\right.
$$


where $\delta$ is a fixed positive time increment. We have immediately from (5.14)

$$
d q^{\prime}\left(t^{\prime}-\delta\right):=q^{\prime}\left(t^{\prime}\right)-q^{\prime}\left(t^{\prime}-\delta\right)=-d q(t)=,-(q(t+\delta)-q(t))
$$

But $d q(t)$ is assigned from eq. (5.13); in particular, we can use the corresponding so-called "backward representation" to write

$$
d q^{\prime}\left(t^{\prime}-\delta\right)=-d q(t)=-\left\{-u(q(t)) d t+\nu^{\frac{1}{2}} d w_{*}(t)\right\}
$$

where we have exploited the relation $\beta_{-}=-\beta_{+}$holding in this particular stationary case [10], and where $w_{*}$ is a standard reversed Wiener process, that is a process which has all properties of an usual Wiener process except from the fact that its increments are independent of the $\sigma$-algebra generated by the future of $w_{*}(t)$. Let us introduce

$$
w^{\prime}\left(t^{\prime}\right)-w^{\prime}\left(t^{\prime}-\delta\right):=w_{*}(t)-w_{*}(t+\delta)
$$

From the definitions and the properties of $w_{*}$, one can immediately see that $w^{\prime}\left(t^{\prime}\right)$ is a standard Wiener process (which, loosely speaking, "goes forward in time"). By inserting eq. (5.17) in eq. (5.16), we are able to write, after standard manipulations, a stochastic differential equation for the diffusion $q^{\prime}$, namely

$$
d q^{\prime}(-t)=u\left(q^{\prime}(-t)\right) d t+\nu^{\frac{1}{2}} d w^{\prime}(-t), \quad-t \in[-T, 0], d t>0 .
$$

Eq. (5.18) provides a possible forward description of what we have called a "specular diffusion". Now, all of these considerations can be extended to the stationary kinematics in comoving coordinates. We can thus conclude that to every solution of the Klein-Gordon equation with positive $\tilde{J}^{0}$ there corresponds a solution where $t$ is replaced by $-t$. The first one is stochastically represented by a stationary diffusion in the comoving coordinates (with respect to the invariant parameter $t$ ), while the second one corresponds to the "rare event" of a "specular diffusion". Changing the parameter $t$ into $-t$ simply leads to a change of sign in $\tilde{S}$, as one can easily see recalling eq. (4.30) and the fact that $\tilde{S}$ is a scalar by construction. Then $\tilde{J}^{0}$ becomes negative and consequently 


$$
J^{0}=\Lambda_{\mu}^{0} \tilde{J}_{*}^{\mu}=-\frac{m c p}{\sqrt{1-\frac{v^{2}}{c^{2}}}}
$$

is also negative at every point. This furnishes us with the desired interpretation.

Having clarified how different subsets of solutions to Klein-Gordon equation can receive different stochastic interpretation, we are now in position to study the non relativistic limit described by Schrödinger equation. Since the latter represents the evolution equation for the quantum state of a single standard spinless particle, we need to consider only those solutions which satisfy conditions (5.10); in addition, we must also select those for which $\|v\| \ll c$. We expect that these last solutions approximately solve dynamical equations equivalent to the Schrödinger one.

The simplest starting point is the Klein-Gordon equation written in cartesian coordinates $\left\{x^{\mu}\right\}:$

$$
\left[\eta^{\mu \nu} \frac{\partial}{\partial x^{\mu}} \frac{\partial}{\partial x^{\nu}}-\frac{m^{2} c^{2}}{\hbar^{2}}\right] \phi(x)=0, \quad \phi(x):=\sqrt{p(x)} \exp \left\{\frac{i}{\hbar} S(x)\right\}
$$

being $p(x)$ a positive normalized probability density over $\mathbf{M}^{4}$. If we define

$$
V_{\mu}:=\frac{1}{m} \frac{\partial}{\partial x^{\mu}} S(x)
$$

then, in agreement also with (5.11), there exists a velocity field $v$ on $\mathrm{R}^{3}$ such that

$$
V^{0}=\frac{c}{\sqrt{1-\frac{v^{2}}{c^{2}}}} \quad, \quad V^{i}=\frac{v^{i}}{\sqrt{1-\frac{v^{2}}{c^{2}}}}
$$

We also construct the four-dimensional osmotic velocity by

$$
U_{\mu}:=\frac{\hbar}{2 m} \frac{\partial}{\partial x^{\mu}} \ln p(x)
$$

The Klein-Gordon equation (5.19) can be now expressed in terms of $U$ and $V$ : by following, in reversed order, the same steps leading from eqs. (4.31) and (4.32) to eq. (4.33), the real part and the gradient of the imaginary part constitute a pair of four-dimensional hydrodynamical equations: 


$$
\left\{\begin{array}{l}
-\left(\frac{\hbar^{2}}{2 m} \eta^{\mu \nu} \frac{\partial}{\partial x^{\mu}} \frac{\partial}{\partial x^{\nu}} U+\hbar U^{\mu} \frac{\partial}{\partial x^{\mu}} U\right)+\hbar V^{\mu} \frac{\partial}{\partial x^{\mu}} V=0 \\
\frac{\partial}{\partial x^{\mu}}\left(p V^{\mu}\right)=0
\end{array}\right.
$$

Starting from (5.23), we now claim that the non relativistic limit is recovered, after substituting $V^{0}$ and $V^{i}$ in terms of (5.21), by taking the limit $\|v\| / c \ll 1$ in the resulting expression, having care to treat as negligible all terms which are multiplied by $\hbar^{2} / c$ and $\hbar^{2} / c^{2}$

The proof goes as follows: let us denote by $\hat{u}$ the spatial part of $U$ and by $v$ the threedimensional velocity field associated to $V$, and let $\hat{t}$ be the time parameter in the inertial frame associated with $\left\{x^{\mu}\right\}$. We put, as usual, $d x^{0}:=c d \hat{t}$. Letting $\|v\| / c \ll 1$, the fourdimensional continuity equation can immediately be rewritten as

$$
\partial_{t} p+\nabla \cdot(p v)=0
$$

The second hydrodynamical equation is now considered in two steps. Separation of the spatial part leads to the following three-dimensional equation:

$$
\begin{aligned}
-\left[\frac{\hbar}{2 m} \nabla^{2} \hat{u}-\frac{\hbar}{2 m} \frac{\partial^{2} \hat{u}}{\partial x^{0^{2}}}\right. & \left.+(\hat{u} \cdot \nabla) \hat{u}+\left(U^{0} \frac{\partial}{\partial x^{0}}\right) \hat{u}\right] \\
& +\left(\frac{v}{\sqrt{1-\frac{v^{2}}{c^{2}}}} \cdot \nabla\right) \frac{v}{\sqrt{1-\frac{v^{2}}{c^{2}}}}+\left(\frac{c}{\sqrt{1-\frac{v^{2}}{c^{2}}}} \frac{\partial}{\partial x^{0}}\right) \frac{v}{\sqrt{1-\frac{v^{2}}{c^{2}}}}=0 .
\end{aligned}
$$

If, as before, we rewrite $d x^{0}=c d \hat{t}$ and take into account the explicit expression of $\hat{U}(5.22)$, we can neglect the second and fourth term, both of which depend on $\hbar^{2} / c^{2}$. In the limit $\|v\| \ll c$, Eq. (5.24) becomes then

$$
-\left[\frac{\hbar}{2 m} \nabla^{2} \hat{u}+(\hat{u} \cdot \nabla) \hat{u}\right]+(v \cdot \nabla) v+\frac{\partial v}{\partial \hat{t}}=0 .
$$

This, together with the continuity equation, is nothing but the hydrodinamical form of Schrödinger equation, which we have also quoted in (4.2). The temporal component of the dynamical equation (5.23) gives rise instead to the following one-dimensional relation:

$$
\begin{aligned}
-\left[\frac{\hbar}{2 m} \nabla^{2} U_{0}-\frac{\hbar}{2 m} \frac{\partial^{2} U_{0}}{\partial x^{0^{2}}}\right. & \left.+\left(U^{0} \frac{\partial}{\partial x^{0}}\right) U_{0}+\left(U^{i} \frac{\partial}{\partial x^{i}}\right) U_{0}\right] \\
+ & \left(\frac{v}{\sqrt{1-\frac{v^{2}}{c^{2}}}} \nabla\right) \frac{c}{\sqrt{1-\frac{v^{2}}{c^{2}}}}+\left(\frac{c}{\sqrt{1-\frac{v^{2}}{c^{2}}}} \frac{\partial}{\partial x^{0}}\right) \frac{c}{\sqrt{1-\frac{v^{2}}{c^{2}}}}=0 .
\end{aligned}
$$


The last two terms are readily recognized to be neglegible in the limit $\|v\| / c \ll 1$. Recalling the explicit form of $U_{0}$ coming from (5.22), we see that the other terms depend on $\hbar^{2} / c$. This proves the assertion.

It is worth to note that the quantum non relativistic limit appears as an approximation of the correct relativistic dynamical equations from a twofold point of view: firstly, the approximation $\|v\| \ll c$ corresponding to the classical non relativistic limit is done; secondly, terms depending on $\hbar^{2} / c$ are also neglected. This fact is perhaps a little unexpected and conceptually intriguing.

Furthermore, an important point of this approach needs to be stressed here, namely the fact that in the relativistic framework no diffusion process is supposed to exist for the inertial observer. A simple probabilistic interpretation is consequently lost in a generic inertial frame. This difficulty may be considered, in a sense, as the stochastic counterpart of non trivial questions which, in the framework of canonical quantization, are related to a consistent definition of observable quantities, such as position operator (see [7] and [19] for any detail). In our procedure, the reason for this fact is that, as observed above, the Wiener process has sample paths which can "go outside" the light cone, so that there exist inertial frames where such spacelike trajectories would appear as going forward and backward in time.

On the other hand, such a peculiarity disappears in the non relativistic limit. As a consequence, the usual Nelson's quantization becomes consistent with the proposed procedure in a non relativistic setting.

\section{DISCUSSION AND OUTLOOK}

The first observation to be done is that stochastic quantization of the free spinless relativistic particle based on comoving (local or global) coordinates is very clean both from a mathematical and a physical point of view. In fact, on one side all probabilistic objects are 
well defined; on the other side, once given stochastic kinematics in the comoving coordinates, dynamical equations naturally emerge from a stochastic version of the Einstein action, by means of the stationary action principle. No additional ad hoc assumptions are necessary, at variance with the path integral approach [18] and previous attempts within stochastic frameworks which we have already quoted.

We also observe that the stochastic quantization procedure proposed in this work gives only to the subset of solutions to Klein-Gordon equation with positive energy and normalized four-current the physical meaning of representing the quantum evolution of one single free relativistic spinless particle. As is well known, this is a consistency property which is not easily achieved within standard canonical (first) quantization [19].

On the other side it may be interesting that one can give a probabilistic interpretation also to other solutions of Klein-Gordon equation, in particular to those with negative energy in terms of specular diffusions as "rare events".

It may also be worthwhile to insist on the fact that the comoving coordinates approach allows to more properly understand how to handle covariant and non covariant quantities. Dynamical equations (which do not contain stochastic terms) are Lorentz covariant, while Markovian kinematics is not: in fact, the latter is covariant only with respect to reparametrizations on $\Sigma_{O}$ and $\gamma_{O}$.

We point out that global comoving coordinates approach introduces a description of the motion of the particle which is well defined on the whole $\mathrm{M}^{4}$ (this in particular provides the technical advantage of eliminating all spurious boundary terms which come out in the local derivation [20]). Moreover, it directly leads to a dynamical theory which is covariant with respect to arbitrary changes of space-time coordinates.

Indeed the results presented in this paper look suitable for the extension to the description of a quantum particle subjected to a gravitational (classical) field. In this case one can furthermore conceive that, in presence of strongly peaked gravitational fields, the degeneracy among the energy levels of the four-dimensional dynamical equation (4.33) breaks down. This is an aspect of the outlined stochastic quantization procedure which deserves further 
investigation [21].

From another point of view, it would be also appealing both on mathematical and physical grounds to consider a sort of second stochastic quantization, by reinterpreting the probability density in the comoving coordinates as a physical scalar field in a space-time dependent random medium. This could in fact be done by exploiting suitable probabilistic techniques (see Ref. 222 for an excellent review of this subject).

\section{ACKNOWLEDGMENTS}

We wish to thank Roberto Onofrio for very useful remarks. 


\section{REFERENCES}

[1] R. M. Dudley, Ark. f. Mat. 6, 241 (1967).

[2] R. Hakim, J. Math. Phys. 9, 1805 (1968).

[3] F. Guerra and P. Ruggiero, Lett. Nuovo Cimento 23, 529 (1978).

[4] D. Dohrn and F. Guerra, Phys. Rev. D 31, 1408 (1985).

[5] M. Serva, Ann. Inst. Henri Poincarè 49, 415 (1988).

[6] P. N. Kaloyerou and J. P. Vigier, J. Phys. A 22, 663 (1989).

[7] G. F. De Angelis, J. Math. Phys. 31, 1408 (1990).

[8] G. F. De Angelis and M. Serva, Ann. Inst. Henri Poincarè 53, 301 (1990).

[9] E. Nelson, Proceedings of the International Workshop on Probabilistic Methods in Mathematical Physics, edited by F. Guerra, M. I. Loffredo and C. Marchioro (World Scientific, Singapore, 1992).

[10] E. Nelson, Quantum Fluctuations (Princeton U. P., Princeton, NJ, 1984).

[11] E. Carlen, Commun. Math. Phys. 94, 293 (1984).

[12] L. M. Morato, Phys. Lett. A 154, 327 (1991); L. M. Morato, Proceedings of the International Workshop on Probabilistic Methods in Mathematical Physics, edited by F. Guerra, M. I. Loffredo and C. Marchioro (World Scientific, Singapore, 1992).

[13] F. De Felice and J. S. Clarke, Relativity on Curved Manifolds (Cambridge U. P., Cambridge, 1990); A. H. Taub, Ann. Rev. Fluid Mech. 10, 301 (1978).

[14] E. C. Zeeman, Topology 6, 161 (1967); see also G. L. Naber, The Geometry of Minkowski Spacetime (Springer, New York, 1992).

[15] F. Guerra and L. M. Morato, Phys. Rev. D 27, 1774 (1983); for a simple survey see 
also L. M. Morato, to appear in Proceedings of the First International Workshop on Quantum-like models and Coherent Effects.

[16] E. Aldrovandi, D. Dohrn and F. Guerra, Acta Appl. Math. 26, 219 (1992).

[17] B. S. DeWitt, Rev. Mod. Phys. 29, 377 (1957).

[18] R. P. Feynman, Rev. Mod. Phys. 20, 267 (1948).

[19] S. S. Schweber, An Introduction to Relativistic Quantum Field Theory (Harper \& Row and John Weatherhill, Inc., Tokyo, 1964).

[20] We notice that the eigenvalue $\mu$ of eq. (4.33) as calculated in the local approach [12] would contain a spurious boundary term which can be made arbitrarily small by suitably restricting the considered neighborhood.

[21] L. M. Morato and L. Viola, in preparation.

[22] S. A. Molchanov, Acta Appl. Math. 22, 139 (1991). 
This figure "fig1-1.png" is available in "png" format from: http://arxiv.org/ps/quant-ph/9505007v1 